\title{
(+)-Catechin Acts as an Infection-Inhibiting Factor in Strawberry Leaf
}

\author{
M. Yamamoto, S. Nakatsuka, H. Otani, K. Kohmoto, and S. Nishimura
}

First, second, and fifth authors: School of Agriculture, Nagoya University, Nagoya 464-8601, Japan; third and fourth authors: Faculty of Agriculture, Tottori University, Tottori 680-8553, Japan.

Current address of M. Yamamoto: Faculty of Agriculture, Okayama University, Okayama 700-8530, Japan.

Current address of S. Nakatsuka: Faculty of Agriculture, Gifu University, Gifu 501-1193, Japan.

S. Nishimura is deceased.

Accepted for publication 11 January 2000.

\begin{abstract}
Yamamoto, M., Nakatsuka, S., Otani, H., Kohmoto, K., and Nishimura, S. 2000. (+)-Catechin acts as an infection-inhibiting factor in strawberry leaf. Phytopathology 90:595-600.

An infection-inhibiting factor (IIF) was isolated from strawberry leaves and identified as (+)-catechin. This compound inhibited the formation of infection hyphae from appressoria of Alternaria alternata, but allowed

response to inoculation with nonpathogenic spores of A. alternata. The accumulation of (+)-catechin on a susceptible host was not induced, however, by inoculation with pathogenic spores of the strawberry pathotype or by inoculation with nonpathogenic spores supplemented with host-specific toxin (AF-toxin I). These results imply that (+)-catechin acts as a protective agent during induced resistance and that AF-toxin I acts as a fungal suppressor of induced resistance.
\end{abstract} both spore germination and appressorial formation. It is a normal component of strawberry leaves, but further accumulates as the major IIF in

Alternaria black spot of strawberry is caused by the strawberry pathotype of Alternaria alternata, which produces host-specific toxins (HSTs) called AF-toxins (16). The name of the strawberry pathotype of A. alternata has been applied since its identification by species name, because the conidial size of this pathogen falls statistically within the range described for A. alternata and the host range of this pathogen is due to the production of HST AFtoxins $(21,22,24,30,31)$. In addition, the application of the pathotype system has been supported by molecular analysis of nuclear ribosomal DNA and mitochondrial DNA (12-14). Under laboratory conditions, the strawberry pathotype is also pathogenic to Japanese pear cultivars susceptible to the Japanese pear pathotype of A. alternata. AF-toxins consist of three active molecules, AF-toxins I, II, and III. These toxins all have a trienoic acid with a terminal epoxide $(19,20)$. The Japanese pear pathotype of A. alternata produces an AK-toxin that is also reported to have these components (18). AFtoxin I is toxic to both susceptible strawberry and Japanese pear cultivars, AF-toxin II is toxic to susceptible Japanese pear cultivars, and AF-toxin III is toxic to susceptible strawberry cultivars. AFtoxin I is thought to be the major of the three AF-toxins because its toxicity to both strawberry and Japanese pear cultivars is consistent with the host range of the strawberry pathotype. The physiological changes in the host cells induced by AF-toxin I and AK-toxin are also very similar $(25,33)$. HSTs are known to induce necrosis and other physiological changes only in susceptible hosts $(21,27)$. It has generally been believed that the role of HSTs in pathogenicity is to induce necrosis, which was thought to be a prerequisite for infection by these fungi $(23,25,35)$. However, it has been observed more recently that infection can take place in the absence of necrosis $(11,33)$. This has led us to hypothesize that the primary role of the toxin is to suppress the resistant reaction of the host plants $(11,22)$.

In strawberry plant-strawberry pathotype interactions, we have previously shown that HSTs play an important role in the suppression

Corresponding author: M. Yamamoto; E-mail address: myama@cc.okayama-u.ac.jp

Publication no. P-2000-0403-02R

(C) 2000 The American Phytopathological Society
Additional keywords: Alternaria black spot of strawberry.

of host resistance and that the treatment of susceptible hosts with HSTs induces susceptibility to nonpathogenic Alternaria spp. $(4,33)$. However, antifungal compounds such as phytoalexins, which might be produced as a result of a resistance response in plant tissue, have not been identified in these pear and strawberry cultivars. Thus, we have been unable to demonstrate a suppression of the resistant reaction by HSTs directly in relation to the accumulation of phytoalexin. Wattad et al. (32) suggested that epicatechin in avocado skin may facilitate resistance to Colletotrichum gloeosporioides in immature fruits through the inhibition of maceration enzymes. We report here that (+)-catechin produced in strawberry leaves acts as a protective agent for fungal infection; that is, as an infectioninhibiting factor (IIF). Also reported is the accumulation of (+)catechin in leaves in response to nonpathogenic A. alternata and the suppression of its accumulation by AF-toxin I produced by the strawberry pathotype of A. alternata.

\section{MATERIALS AND METHODS}

Plants. Strawberry plants (Fragaria $\times$ ananassa) cvs. Morioka16 and Hokowase, which are susceptible and resistant, respectively, to Alternaria black spot (16), were grown in a greenhouse with a controlled temperature between 18 and $28^{\circ} \mathrm{C}$ (minimum 12-h day). Detached young leaves were used unless otherwise noted. More than 80 susceptible strawberry plants were used to harvest $1,000 \mathrm{~g}$ of fresh induced leaves for $(+)$-catechin isolation.

To prepare aseptic cv. Morioka-16 plants, aseptic mericlones were obtained from runners and grown on Murashige and Skoog (MS) basal medium (Sigma-Aldrich, St. Louis) with $1.5 \%$ agar under aseptic conditions. Plants that showed vigorous growth and looked normal in appearance were transferred into sterile 1,000-ml culture bottles containing solid growth medium $(\times 0.5 \mathrm{MS}$ basal salts) with $1.5 \%$ agar. The bottles were sealed with aluminum foil and maintained in a growth chamber $\left(25^{\circ} \mathrm{C}\right.$ during the day and $18^{\circ} \mathrm{C}$ at night, 18-h day length). Four plants showing similar growth rates were selected from among the aseptic plants 1 month after transfer. Plants were 10 to $15 \mathrm{~cm}$ in height and had eight trifoliate leaves 
each. None of the plants showed any variation in sensitivity to AFtoxin I resulting from the tissue culture. Two plants were then maintained in aseptic conditions, while the other two were exposed to air by replacing the sealing foil with foil containing a 3-mm pore. Sterilized distilled water was added to the control aseptic plants to maintain saturated humidity. Strawberry plants are trifoliate; therefore, a newly developed shoot was chosen and one of the three leaflets harvested to examine the content of $(+)$-catechin before treatment (day 0). A second leaflet was harvested after 1 week (week 1) and the last harvested a week later (week 2). Harvested leaves were immediately weighed and examined to quantify the (+)-catechin content. The harvest of leaflets from aseptic plants was performed on a clean bench. Throughout the experiment, harvested leaflets from both aseptic and nonaseptic plants showed no visible symptoms of disease, and no apparent injury resulted from incubation.

Fungi and AF-toxin I. The pathogenic strain O-187 and the nonpathogenic strain O-94 of A. alternata were used in this experiment. These strains were grown in potato sucrose broth (PSB; $200 \mathrm{ml}$ of extract from boiled potatoes, $20 \mathrm{~g}$ of sucrose, and $800 \mathrm{ml}$ of water) for 14 days at $27^{\circ} \mathrm{C}$. Purified AF-toxin I was prepared from culture filtrates of $\mathrm{O}-187$ as described previously $(16,20)$ and used in an aqueous solution at $2 \times 10^{-7} \mathrm{M}$. Spores formed on mycelial mats on the PSB and were used for inoculation. Spores, suspended in distilled water at $5 \times 10^{5}$ spores per $\mathrm{ml}$, were sprayed onto leaf surfaces, the epidermis of onion bulbs, and cellophane tubes (Visking tube; Wako Pure Chemical Inc., Osaka, Japan) on glass slides and then incubated in a moist chamber for $24 \mathrm{~h}$ at $27^{\circ} \mathrm{C}$. Spots on the leaves caused by the pathogen were counted and a transparent leaf segment $(1 \times 1 \mathrm{~cm})$ prepared from the leaf using a solution of lactic acid/phenol/distilled water $(4: 1: 1, \mathrm{vol} / \mathrm{vol} / \mathrm{vol})$ with $0.1 \%$ cotton blue. Microscopic observation of the inoculated surface was then undertaken. Spore germination, germ tube elongation, appressorium formation per germ tube, and infection hyphae formation per appressorium were observed under a microscope. Cladosporium fulvum strain 1, which causes tomato scab, was grown on potato sucrose agar plates to produce spores.

Induction and bioassay of IIF in strawberry leaf. For the induction of resistance, strawberry leaves were inoculated with a spore suspension of nonpathogenic A. alternata at $1 \times 10^{6}$ spores per $\mathrm{ml}$ of distilled water. Distilled water was sprayed on uninduced leaves. To compare the degree of induction, the half-leaf method

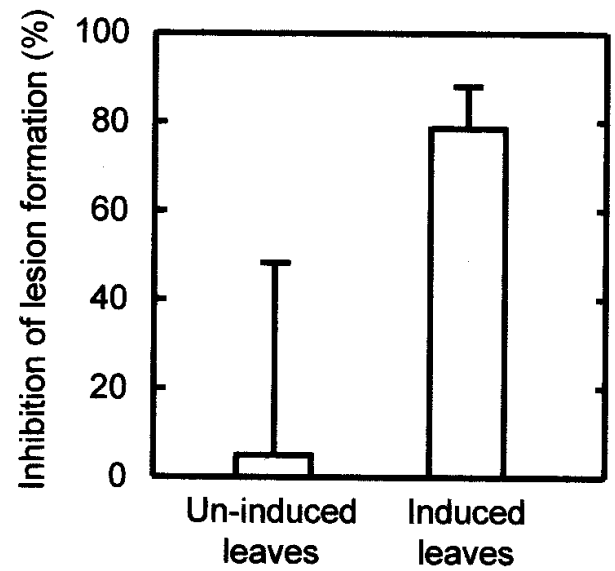

Fig. 1. Induction of infection-inhibiting activity in ethyl acetate extracts of strawberry leaves following inoculation with nonpathogenic spores. Ethyl acetate extracts were obtained from susceptible strawberry leaves (cv. Morioka16) inoculated with nonpathogenic spores of Alternaria alternata (induced) or sprayed with distilled water (uninduced). Infection-inhibiting activity was detected when the extracts were applied to inoculation of pathogenic spores of $A$. alternata on the susceptible leaves. Inhibition is shown as a percentage of each water control ( $0 \%$ inhibition). Each experiment contained four replications and two independent experiments were performed. Bars indicate standard deviations. was used. Two leaves were used to prepare one sample. One half of each leaf was induced, whereas the opposite half was treated with distilled water. In a time course experiment, in which the application of the half-leaf method seemed to be difficult, each leaf was cut into four pieces and then all the pieces were inoculated with nonpathogenic spores, pathogenic spores, or sprayed with distilled water. A piece of the leaf was harvested to compare between $0 \mathrm{~h}$ and the specific incubation period. In the case of large-scale preparation, the induction was carried out on $250 \mathrm{~g}$ of fresh strawberry leaf at once, and four separate experiments produced a total of extracts from $1,000 \mathrm{~g}$ of induced leaf. After $24 \mathrm{~h}$ of incubation at $27^{\circ} \mathrm{C}$ to allow induction of resistance, strawberry leaves were immediately homogenized in $70 \%$ ethanol ( $5 \times$ volume), and the homogenates were filtered. Midribs of the leaves were cut off before ethanol extraction, except for large-scale preparation. The ethanol extracts were evaporated under reduced pressure at $40^{\circ} \mathrm{C}$ until all of the ethanol was removed, and then the resultant aqueous solution was adjusted to $\mathrm{pH} 3.0$ with $0.2 \mathrm{M} \mathrm{HCl}$ and extracted with ethyl acetate. The ethyl acetate extracts were evaporated under reduced pressure at $40^{\circ} \mathrm{C}$. The residues were then redissolved in distilled water of equivalent weight to the starting weight of the extracted leaf. For example, when a sample was extracted from $1 \mathrm{~g}$ fresh weight of leaf, the material at each step of the purification was dissolved in $1 \mathrm{ml}$ of distilled water. In the case of lipophilic samples, a small percentage of acetone $(<5 \%)$ was used to redissolve the residue, and the same percentage of acetone was added to the distilled water control.

To enable the bioassay of the infection-inhibiting activity, spores of the pathogenic strain were suspended in an aqueous solution and prepared as described above, after which the resulting suspension was sprayed onto halves of susceptible strawberry leaves. Adjacent halves of the leaves acted as controls. These were inoculated with spores of the pathogenic strain suspended in distilled water. Each bioassay was carried out with a set of four replications by the half-leaf method and repeated twice. Spots appearing on both halves were counted, and then spore germination, appressorial formation, and formation of infection hyphae were observed on the transparent sections under a microscope to determine which steps of the infection process were, or were not, inhibited. Fifty spores per replication were observed and a total of 400 spores counted. Each rate of infection behavior was calculated as follows: rate of germination, by the number of germinated spores per number of spores observed; rate of appressorium formation, by the number of appressorium per number of germinating tubes observed; and rate of infection hypha formation, by the number of infection hypha per number of appressoria observed. Inhibition of infection behavior was shown as the percentage of inhibition relative to the distilled water control ( $0 \%$ inhibition). Lesion formation was based on the number of spots per square centimeter. Inhibition of spot formation, indicating infection-inhibiting activity, was quantified as the percentage of inhibition relative to the distilled water control ( $0 \%$ inhibition). The inoculation on the epidermis of onion bulbs and the cellophane tube was used only to observe fungal infection behavior to ensure that infection-inhibiting activity was derived from IIF itself without any interactions with other compounds in strawberry cells. Cladosporium fulvum was employed in the screening process for antifungal compounds, and spore germination and germ tube elongation on glass slides were observed under the microscope. A total of 100 spores was observed to calculate the rate of germination and 100 germ tubes were measured to calculate the germ tube elongation in one experiment. Inhibition of germination and germ tube elongation was quantified as the percentage of inhibition relative to the distilled water control ( $0 \%$ inhibition).

Purification of IIF from strawberry leaf. In preliminary studies and the time-course experiments, the ethyl acetate extracts of strawberry leaves were separated by silica gel thin-layer chromatography (TLC) (Silica gel $60 \mathrm{~F}_{254}$; E. Merck AG, Darmstadt, Germany) using a chloroform/methyl alcohol (3:1, vol/vol) solvent. 
The silica gel layer was divided into nine fractions according to $R_{f}$ value, and compounds in each fraction were eluated from the gel with methanol. The methanol was then removed under vacuum and the residue dissolved in distilled water. Residues were then bioassayed by the pathogen and strawberry leaf system to detect IIFs and by using spores of Cladosporium spp. on glass slides to detect antifungal compounds.

In a large-scale preparation, the ethyl acetate extracts were purified by open silica gel column chromatography (Wako gel C-200; Wako Pure Chemical Inc.) with the following solvents: hexane-ethyl acetate (80 to $100 \%$ ethyl acetate), ethyl acetate-methanol (0 to $20 \%$ methanol), chloroform-ethyl acetate ( 75 to $100 \%$ ethyl acetate), and chloroform-methanol (5 to $10 \%$ methanol, twice).

To quantify the (+)-catechin content, ethanol extracts were also separated by TLC, as described above. Under UV light (254 nm), a zone corresponding to the (+)-catechin region was visualized as a quenching spot with an identical $R_{f}$ value to that of reagent (+)catechin (Sigma-Aldrich) applied to a lane adjacent to the sample. Methanol was used to elute (+)-catechin from the scraped gel. Measurements of the extracted (+)-catechin concentration were then carried out using reagent $(+)$-catechin as a standard, with absorbance at $280 \mathrm{~nm}$, which is $\lambda_{\max }\left(\mathrm{CH}_{3} \mathrm{OH}\right)$ of (+)-catechin.

\section{RESULTS}

Characterization of IIF from strawberry leaves. In the survey of IIF, ethyl acetate extracts of induced and uninduced leaf halves were examined to see if lesion formation was inhibited. The extracts of uninduced halves had little inhibition of lesion formation but a considerably large variance compared with that of induced halves. A significant increase in inhibition of lesion formation was detected in extracts of induced halves by inoculation with nonpathogenic spores (Fig. 1). From these results, we assumed that IIF might preexist at the lower content or that IIF was preinduced by environmental factors during greenhouse planting. Direct separation of the extracts by TLC showed that IIFs that moved near the solvent front on TLC-inhibited germ tube elongation of Cladosporium fulvum (Fig. 2) but did not inhibit germination nor germ tube elongation of $A$. alternata (data not shown). Although preliminary separation of ethanol extracts by TLC indicated several IIFs, one IIF ( $R_{f}$ value of 0.22 to 0.33$)$ predominated. The others

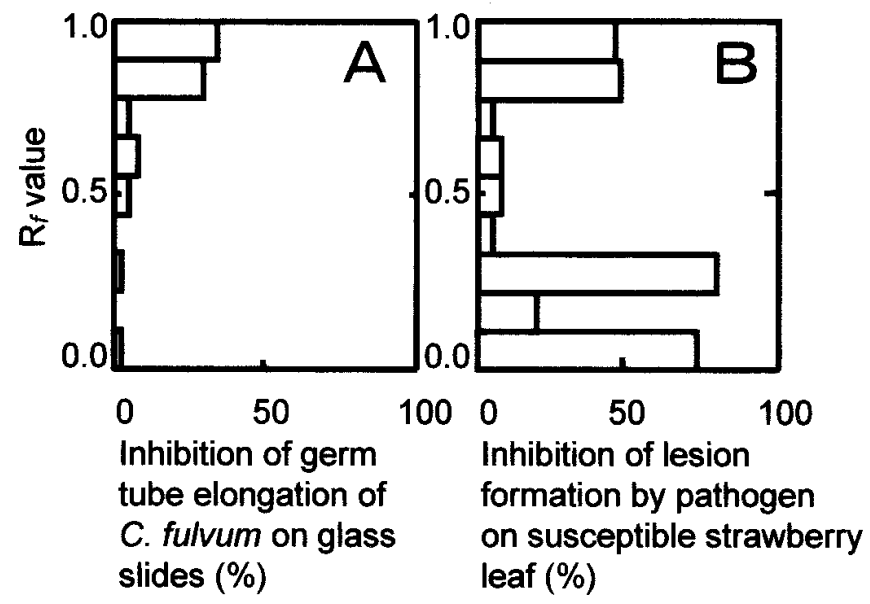

Fig. 2. A typical separation of active fractions in leaf extracts on silica gel thin-layer chromatography (TLC). Ethyl acetate extracts of resistance-induced susceptible strawberry leaves were separated on TLC and were examined to determine inhibition of $\mathbf{A}$, germ tube elongation and $\mathbf{B}$, lesion formation. Spores of Cladosporium fulvum were germinated on glass slides to detect antifungal activity, whereas spores of the Alternaria alternata strawberry pathotype were inoculated onto host leaves with each eluent. Inhibition is shown as a percentage of each water control ( $0 \%$ inhibition). Two independent experiments showed a similar chromatogram. proved to be mixtures of more than two minor IIFs. Purification of the predominant IIF was examined in a large scale using extracts of induced leaves. A total of $160 \mathrm{mg}$ of the chloroform-insoluble compound was recovered from $1,000 \mathrm{~g}$ fresh weight of strawberry leaves. Partial chemical structures determined from ${ }^{1} \mathrm{H}-\mathrm{NMR}$ and ${ }^{13} \mathrm{C}-\mathrm{NMR}$, combined with data on the mass spectrum $\left(\mathrm{C}_{15} \mathrm{H}_{14} \mathrm{O}_{6}\right)$, suggested that this compound was a catechin. It was then confirmed as (+)-catechin by comparison of spectrum data between the purified extract and the commercially available catechins.

Inhibitory activity of (+)-catechin on infection behavior of the pathogen. Purified (+)-catechin evidently inhibited the formation of infection hyphae and subsequent lesion formation on susceptible strawberry leaves, rather than spore germination and appressorial formation (Fig. 3). Formation of infection hyphae was slightly inhibited at $100 \mu \mathrm{g} / \mathrm{ml}\left(3.4 \times 10^{-4} \mathrm{M}\right)$ and further inhibited with increasing concentrations of $(+)$-catechin. Authentic reagent $(+)$-catechin caused a similar dose-response inhibition (data not shown). To assess the possibility that (+)-catechin induces a resistant response in strawberry leaf, the epidermis of the onion bulb, which is a nonhost, was used as a model epidermis. Although spores of the pathogen could penetrate into epidermal cells of the onion bulb, penetrations were markedly reduced in the presence of (+)-catechin at $500 \mu \mathrm{g} / \mathrm{ml}$. The rates of spore germination and appressorium formation were not lowered by the treatment of $(+)$ catechin when compared with that of the distilled water control (data not shown).

Accumulation of (+)-catechin in strawberry leaf and its suppression by AF-toxin I. Infection hyphae of $A$. alternata were produced within 6 to $8 \mathrm{~h}$ of inoculation, and the maximum accumulation of $(+)$-catechin occurred within $8 \mathrm{~h}$. When nonpathogenic spores were used for inoculation, (+)-catechin accumulated to an inhibitory concentration (Fig. 4) and the actual accumulation reached $513 \pm 50 \mu \mathrm{g} / \mathrm{g}$ fresh leaf weight. (+)-Catechin preexisted at $225 \pm$ $105 \mu \mathrm{g} / \mathrm{g}$ fresh leaf weight; therefore, the accumulation increased more than twofold. The inhibition of the accumulated (+)-catechin in strawberry leaves (47\% inhibition) was consistent with the inhibition of purified (+)-catechin, $50 \%$ at $500 \mu \mathrm{g} / \mathrm{ml}$ (Fig. 3). In this, a sample from $1 \mathrm{~g}$ of leaf was dissolved in $1 \mathrm{ml}$ of distilled

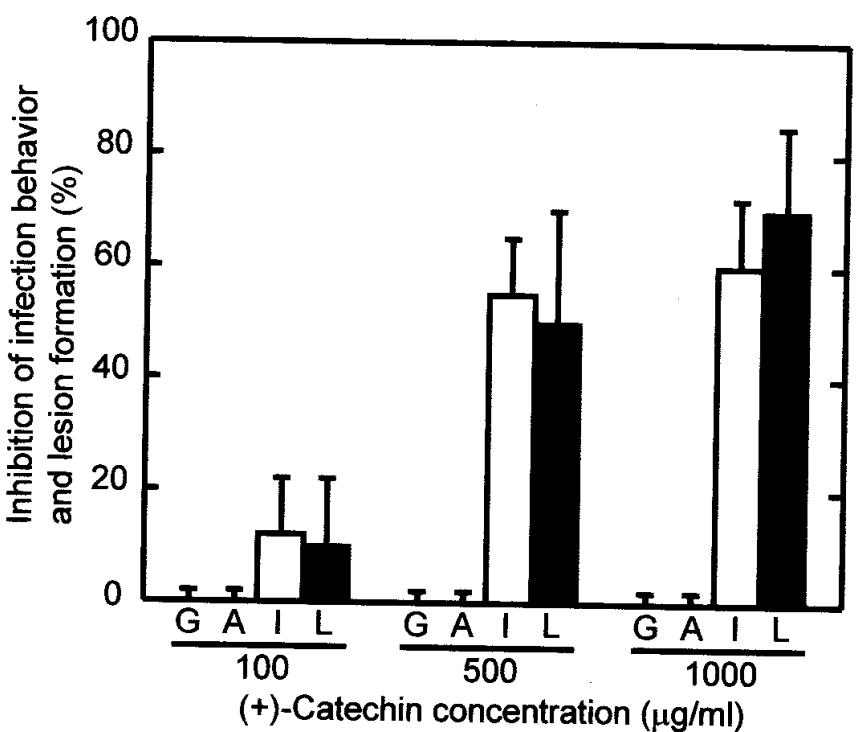

Fig. 3. Effect of (+)-catechin on infection behavior of pathogenic spores on susceptible strawberry leaf. Spores in (+)-catechin solution and in distilled water (control) were inoculated. After $24 \mathrm{~h}$ of incubation, rates of germination (G), appressorium formation (A), infection hypha formation (I), and lesion formation per square centimeter (L) were calculated. Each inhibition against controls ( $0 \%$ inhibition) is shown. $\mathrm{G}$ and $\mathrm{A}$ were not inhibited any concentration of $(+)$-catechin; therefore, no column indicating an average of inhibition percentage is shown. Bars indicate standard deviations. Each bioassay contained four replications and was repeated twice. 
water according to the assay condition. Distilled water did not cause $(+)$-catechin accumulation. Inoculation with pathogenic spores or nonpathogenic spores, along with AF-toxin I, did not induce (+)catechin accumulation in susceptible leaves, but did induce the accumulation in resistant leaves.

We used greenhouse-grown strawberry plants in all of these experiments; therefore, the preexistence of (+)-catechin might have been induced by microorganisms on the plants. To test for this possibility, we examined the content and accumulation of (+)-catechin in plants using aseptic plants as models. Under such conditions, we found that $(+)$-catechin existed in aseptic strawberry leaves at 140 $\mu \mathrm{g} / \mathrm{g}$ fresh weight of leaf, which was lower than the $225 \mu \mathrm{g} / \mathrm{g}$ detected in the greenhouse plants (Fig. 5). The weight gains of the leaflets in both aseptic and nonaseptic plants were very similar. We observed small fungal and bacterial growths on the agar medium in bottles of nonaseptic plants. We did not isolate and identify these microorganisms; however, all of the plant parts appeared to be healthy throughout this experiment. Strawberry leaflets that were exposed to air did, however, accumulate (+)-catechin, while the contents of $(+)$-catechin remained almost constant in leaflets that were kept in aseptic conditions.

\section{DISCUSSION}

We report here the accumulation of (+)-catechin as an IIF in response to inoculation with a nonpathogenic strain of A. alternata and the subsequent inhibition of fungal penetration into strawberry leaf tissues. We did not find evidence for phytoalexin-like sub-

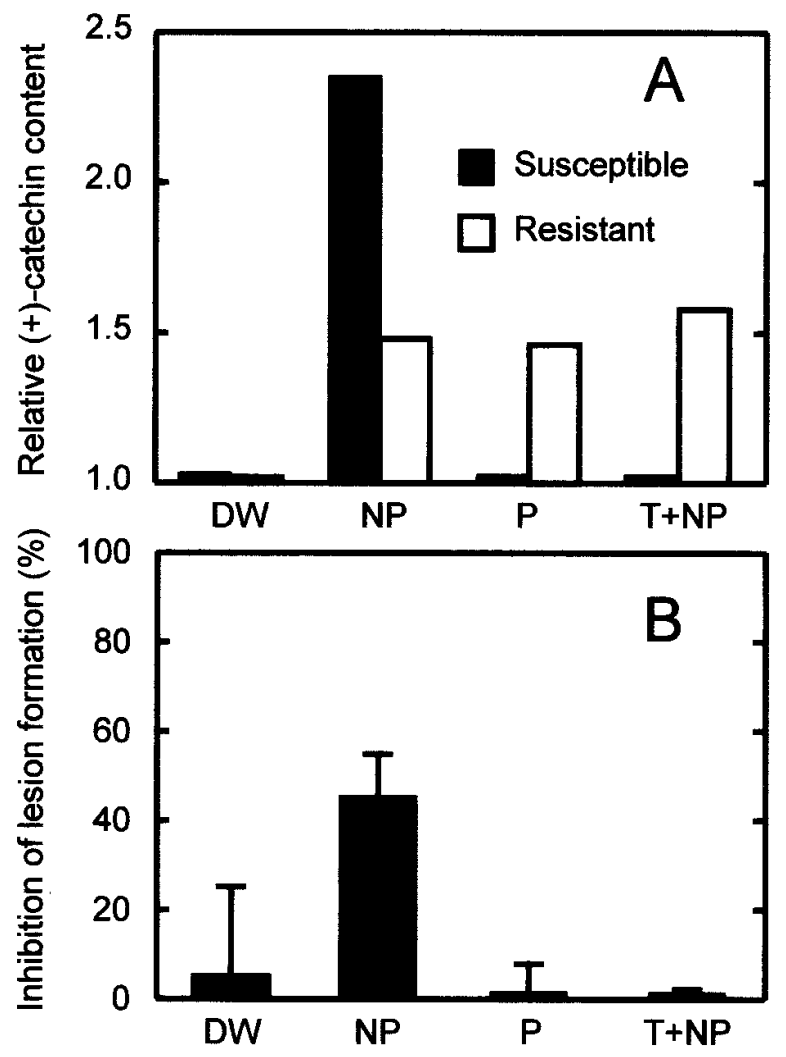

Fig. 4. Differential accumulation of (+)-catechin in strawberry leaf after inoculation. The content of accumulated (+)-catechin in leaves within $8 \mathrm{~h}$ after inoculation with nonpathogenic spores (NP), pathogenic spores (P), or NP suspended in AF-toxin I solution (T+NP) was determined. A, The contents of (+)-catechin are shown as a ratio of the baseline at $0 \mathrm{~h}$. Susceptible (black column) and resistant (white column) leaves were examined. B, Infectioninhibiting activity of (+)-catechin accumulated in susceptible leaves at the time that content was determined was bioassayed using lesion formation (black column). Each experiment contained two replications and was repeated twice. Bars indicate standard deviations. stances that strongly inhibit spore germination or elongation of the germ tube of pathogenic A. alternata. However, germ tube elongation was inhibited following inoculation with Cladosporium fulvum, a species that is often employed to screen for the presence of antifungal activity. These results are consistent with the lack of reports of phytoalexin from strawberry leaves. We concluded that the accumulation of (+)-catechin corresponded with the inoculation of nonpathogenic spores and was a major part of the resistance induced in strawberry leaf. There is still a possibility in vivo that other minor IIFs detected in the initial stages of purification were involved in responses inhibiting fungal penetration together with (+)-catechin.

We have used (+)-catechin accumulation as a measure of induced resistance responses in this strawberry plant-strawberry pathotype of A. alternata interaction. (+)-Catechin preexists at less-thanactive concentrations in strawberry leaf before the induction of resistance by inoculation with nonpathogenic spores. This, together with its rapid accumulation (within $8 \mathrm{~h}$ ) to levels sufficient to inhibit the formation of infection hyphae induced by later inoculation, suggests that (+)-catechin is an inhibitin, as defined by Ingram (8). The accumulation of (+)-catechin was found not only in susceptible strawberry plants but also in resistant strawberry plants, which means this normal component of strawberry leaf is involved in resistant responses. In this interaction, unlike race-cultivar interactions of other diseases, hypersensitive cell death is not observed in resistant responses, because nonpathogenic spores could not invade host cells (33). Furthermore, we could not show the involvement of phytoalexin in this system. These results may support the view that accumulation of (+)-catechin is involved in a resistant mechanism different from those in which phytoalexins and hypersensitive responses are involved; for example, cultivarspecific resistant mechanisms. A proposed general or nonhost resistance may involve accumulation of $(+)$-catechin $(1,6)$. The involvement of IIFs such as (+)-catechin in induced resistance represented here is the first example of such a relationship; therefore, we will need more proof before we can hypothesize about its role in the host-parasite interactions described above. The accumulation of IIF may not be specific to strawberry leaf, however, because 3,5di- $O$-caffeoylquinic acid was recently isolated from the Japanese pear (cv. Nijisseiki) as an IIF (10,26). Furthermore, leaves of pea plants have been shown to contain IIF-like metabolites other than pisatin, a well-known phytoalexin (34). Inhibition of fungal penetration was also detected on the cellophane membrane (data not shown).

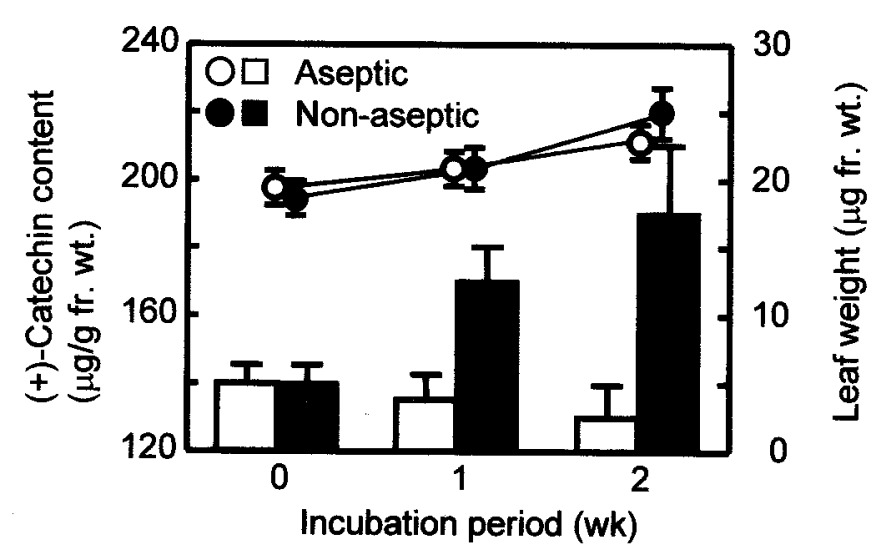

Fig. 5. Accumulation of (+)-catechin in leaves of strawberry plants grown in aseptic conditions and in the air. Germ-free strawberry plants were grown in aseptic conditions and were used to determine both the preexistence and accumulation of (+)-catechin after exposing plants to the air. The fresh weights of the leaves (circles and lines) and the content of (+)-catechin (columns) in the leaves were determined. All four plants were grown in aseptic conditions, and then two of them were further grown in aseptic conditions (white circles and columns), while the others were grown in air (black circles and columns). Bars indicate standard deviations. 
A relatively high concentration of (+)-catechin $(>100 \mu \mathrm{g} / \mathrm{ml})$ was needed to inhibit the formation of infection hyphae from appressoria compared with phytoalexins, and it did not affect spore germination and appressorial formation, even at $1,000 \mu \mathrm{g} / \mathrm{ml}$. Mace et al. (15) discussed the antifungal activity of (+)-catechin on Verticillium spp. development in cotton, whereas, in our study, (+)-catechin was unable to inhibit the growth of either Cladosporium fulvum or A. alternata (data not shown). The mode of action that could explain such specific activity on the formation of infection hyphae is still unknown. A mode of action of flavonoids, including (+)-catechin, may be through some inhibition of penetration enzymes. Wattad et al. (32) discussed the infection-inhibiting activity of epicatechin against Colletotrichum gloeosporioides in the skin of avocado fruits. The existence of epicatechin in the skin of immature avocado fruits and the inhibition of pectate lyase by epicatechin may suggest similar roles and localization of these IIFs. Thus, catechins may be located mainly in the epidermis and act as IIFs. However, (+)-catechin prevented spores of A. alternata from penetrating into epidermal cells of onion and cellophane tubes (data not shown), which were used as model walls to evaluate aggressiveness, as well as host strawberry cells. These results indicated that the action of (+)-catechin does not depend on the materials the pathogenic spores were trying to penetrate. Recent work focusing on fungal penetration from appressoria showed that infection hyphae are formed through a series of well-organized processes that appear to be essential for successful penetration into the host cell (2, $7,17)$. Infection pores or infection pegs from the infection pores are elaborately constructed, and (+)-catechin might have an action on appressoria in these phases of morphological change. However, there are differences in the structure and characteristic formation of appressoria between Alternaria and the other genera involved, namely Colletotrichum and Magnaporthe, and these should be considered in any future study of IIFs (9).

In preliminary experiments, we found that the activity of IIF varied, depending on the leaf examined. The half-leaf method was used for induction experiments, as well as for the bioassay of inhibitory activity of $(+)$-catechin, because further accumulation in addition to preexisting (+)-catechin should reveal induction of a resistant response (Fig. 1). A variable content of (+)-catechin preexisted in leaves at the rate of $225 \pm 105 \mu \mathrm{g} / \mathrm{g}$ fresh weight of leaf and was also reflected as a variance in inhibition of lesion formation by (+)-catechin in the distilled water control (Fig. 4B). On the other hand, different contents of preexisting $(+)$-catechin, which was typically reflected as a variance of uninduced leaves (Fig. 4B), led us to a hypothesis that the accumulation of (+)-catechin in leaf is sensitive to environmental factors. The hypothesis that environmental factors might induce (+)-catechin accumulation may be acceptable, because each individual plant and each individual leaf would be subjected to different strength of actions of environmental factors. We showed increased levels of (+)-catechin after exposing aseptic plants to the air. This indicated that (+)-catechin accumulation may be induced not only by nonpathogenic A. alternata, but also by environmental factors including microorganisms that are abundant in nature. (+)-Catechin, however, may not accumulate simply as a secondary metabolite along with normal growth, because aseptic plants had almost constant levels of (+)-catechin and the variance of them was less than that of uninduced leaves grown in the greenhouse during this experiment. The possibility that naturally growing plants may have already acquired resistance by interaction with these microorganisms has been suggested by Sahashi et al. (28). Alternatively, the cessation of aseptic conditions may cause physiological changes in the plants through changes in the surrounding microenvironment.

Hayashi et al. (5) reported that pathogenic spores of strawberry pathotype produced AF-toxin I at levels of up to $10^{-8} \mathrm{M}$ in the fluid surrounding germinating spores. We employed AF-toxin I at $10^{-7} \mathrm{M}$ and found that it could induce infection of nonpathogenic spores of A. alternata and could also suppress induced resistance, but only in the susceptible cv. Morioka-16 (33). AF-toxin I exhibited complete suppression of de novo accumulation of (+)-catechin after induction only in susceptible leaves. This agrees with our previous reports that AF-toxin I suppressed induced resistance in susceptible strawberry leaf. In this experiment, we found that a resistant cultivar accumulated (+)-catechin in response to inoculation both with and without AF-toxin I, although the induction rate was only $70 \%$ of that demonstrated for the susceptible cultivar. Such specificity is likely to result from specific physiological changes, which we have reported only for susceptible cultivars. Suppression of host resistance in the early pathogenesis of plant-pathogenic fungi has been reported in several other diseases in which causal agents were described as suppressors $(3,29)$. We previously proposed that HSTs could suppress the potential resistance that would be expected to be induced by inoculation with a nonpathogenic strain of A. alternata $(22,33)$. However, in the current study, we were clearly able to show a pathological role of AF-toxin I as a suppressor using the increase of (+)-catechin as an indicator for induced resistance. Thus, AF-toxin I, which could suppress the host defense response including the accumulation of IIF, would allow pathogenic spores to establish an infection in susceptible plants, but not in resistant plants lacking sensitivity to AF-toxin I.

\section{ACKNOWLEDGMENTS}

We thank T. Tsuge for valuable suggestions and discussions.

\section{LITERATURE CITED}

1. Bushnell, W. R., and Rowell, J. B. 1981. Suppression of defense reaction: A model for roles in specificity. Phytopathology 71:1012-1014.

2. Chemley, F. G., and Valent, B. 1990. Genetic analysis of melanin-deficient, non-pathogenic mutants of Magnaporthe grisea. Mol. Plant-Microbe Interact. 3:135-143.

3. Doke, N. 1975. Prevention of the hypersensitive reaction of potato cells to infection with an incompatible race of Phytophthora infestance by constituents of the zoospores. Physiol. Plant Pathol. 7:1-7.

4. Hayami, C., Otani, H., Nishimura, S., and Kohmoto, K. 1982. Induced resistance in pear leaves by spore germination fluids of nonpathogens to Alternaria alternata Japanese pear pathotype and suppression of the induction by AK-toxin. J. Fac. Agric. Tottori Univ. 17:9-17.

5. Hayashi, N., Tanabe, K., Tsuge, T., Nishimura, S., Kohmoto, K., and Otani, H. 1990. Determination of host-selective toxin production during spore germination of Alternaria alternata by high-performance liquid chromatography. Phytopathology 80:1088-1091.

6. Heath, M. C. 1981. A generalized concept of host-parasite specificity. Phytopathology 71:1121-1123.

7. Howard, R. J., Ferrari, M. A., Roachi, D. H., and Money, N. P. 1991. Penetration of hard substrates by a fungus employing enormous turgor pressures. Proc. Natl. Acad. Sci. U.S.A. 88:11281-11284.

8. Ingram, J. L. 1973. Disease resistance in higher plants: The concept of pre-infectional and post-infectional resistance. Phytopathol. Z. 78:314-335.

9. Kawamura, C., Moriwaki, J., Kimura, N., Fujita, Y., Fujii, S., Hirano, T., Koizumim, S., and Tsuge, T. 1997. The melanin biosynthesis gene of $\mathrm{Al}$ ternaria alternata can restore pathogenicity of the melanin-deficient mutants of Magnaporthe grisea. Mol. Plant-Microbe Interact. 10:446-453.

10. Kodama, M., Wada, H., Otani, H., Kohmoto, K., and Kimura, Y. 1998. 3,5-Di-O-caffeoylquinic acid, as infection-inhibiting factor from Pyrus pyrifolia induced by infection with Alternaria alternata. Phytochemistry 47:371-373.

11. Kohmoto, K., Otani, H., Kodama, M., and Nishimura, S. 1989. Can accessibility to fungal invasion be induced by host-specific toxins without necessitating necrotic cell death? Pages 249-265 in: Phytotoxins and Plant Pathogenesis. A. Graniti, A. Ballio, and R. D. Durbin, eds. Springer-Verlag, Berlin.

12. Kusaba, M., and Tsuge, T. 1994. Nuclear ribosomal DNA variation and pathogenic specialization in Alternaria fungi known to produce hostspecific toxins. Appl. Environ. Microbiol. 60:3055-3062.

13. Kusaba, M., and Tsuge, T. 1995. Phylogeny of Alternaria fungi known to produce host-specific toxins on the basis of variation in internal transcribed spacers of ribosomal DNA. Curr. Genet. 28:491-498.

14. Kusaba, M., and Tsuge, T. 1997. Mitochondrial DNA variation in hostspecific toxin-pathogens in the genus Alternaria. Ann. Phytopathol. Soc. Jpn. 63:463-469. 
15. Mace, M. C., Bell, A. A., and Stipanovic, R. D. 1978. Histochemistry and identification of flavonols in Verticillium wilt-resistant and -susceptible cottons. Physiol. Plant Pathol. 13:143-149.

16. Maekawa, N., Yamamoto, M., Nishimura, S., Kohmoto, K., Kuwada, K., and Watanabe, Y. 1984. Studies on host-specific AF-toxins produced by Alternaria alternata strawberry pathotype causing Alternaria black spot of strawberry. (1) Production of host-specific toxins and their biological activities. Ann. Phytopathol. Soc. Jpn. 50:600-609.

17. Mitchell, T. K., and Dean, R. A. 1993. The cAMP-dependent protein kinase catalytic subunit is required for appressorium formation and pathogenesis by the rice blast pathogen Magnaporthe grisea. Plant Cell 7: 1869-1878.

18. Nakashima, T., Ueno, T., Fukami, H., Taga, T., Masuda, H., Osaki, K., Otani, H., Kohmoto, K., and Nishimura, S. 1985. Isolation and structures of AK-toxin I and II, host-specific phytotoxic metabolites produced by Alternaria alternata Japanese pear pathotype. Agric. Biol. Chem. 49: 807-815.

19. Nakatsuka, S., Ueda, K., Goto, T., Yamamoto, M., Nishimura, S., and Kohmoto, K. 1986. Structure of AF-toxin II, one of the host-specific toxins produced by Alternaria alternata strawberry pathotype. Tetrahedron Lett. 27:2753-2756.

20. Namiki, F., Yamamoto, M., Nishimura, S., Nakatsuka, S., Goto, T., Kohmoto, K., and Otani, H. 1986. Studies on host-specific toxins produced by Alternaria alternata strawberry pathotype causing Alternaria black spot of strawberry. (4) Protective effect of pretreatment with AFtoxin II on AF-toxin I induced toxin action and fungal infection against strawberry tissues. Ann. Phytopathol. Soc. Jpn. 52:428-436.

21. Nishimura, S., and Kohmoto, K. 1983. Host-specific toxins and chemical structures from Alternaria species. Annu. Rev. Phytopathol. 21:87-116.

22. Nishimura, S., and Kohmoto, K. 1983. Role of toxins in pathogenesis. Pages 137-157 in: Toxins and Plant Pathogenesis. J. M. Daly and B. J. Deverall, eds. Academic Press Australia, Sydney, Australia.

23. Nishimura, S., and Scheffer, R. P. 1965. Interactions between Helminthosporium victoriae spores and oat tissue. Phytopathology 55:629-634.

24. Nishimura, S., Sugihara, M., Kohmoto, K., and Otani, H. 1978. Two different phases in pathogenicity of the Alternaria pathogens causing black spot of Japanese pear. J. Fac. Agric. Tottori Univ. 13:1-10.

25. Otani, H., Nishimura, S., Kohmoto, K., Yano, K., and Seno, T. 1975. Nature of specific susceptibility to Alternaria kikuchiana in Nijisseiki cultivar among Japanese pear. (V) Role of host-specific toxin in early step of infection. Ann. Phytopathol. Soc. Jpn. 41:467-476.

26. Otani, H., Sase, M., Kohmoto, K., and Nishimura, S. 1982. Accumulation of infection-inhibiting factor in Japanese pear leaf. (In Japanese.) (Abstr.) Ann. Phytopathol. Soc. Jpn. 48:111.

27. Pringle, R. B., and Scheffer, R. P. 1964. Host-specific toxins. Annu. Rev. Phytopathol. 2:133-156.

28. Sahashi, N., Tsuji, H., and Shishiyama, J. 1989. Barley plants grown under germ-free condition have increased susceptibility to two powdery mildew fungi. Physiol. Mol. Plant Pathol. 34:163-170.

29. Shiraishi, T., Oku, H., Yamashita, M., and Ouchi, S. 1978. Elicitor and suppressor of pisatin induction in spore germination fluid of pea pathogen, Mycosphaerella pinodes. Ann. Phytopathol. Soc. Jpn. 44:659-665.

30. Watanabe, Y., and Umekawa, M. 1977. A new disease of strawberry caused by Alternaria sp. (In Japanese.) (Abstr.) Ann. Phytopathol. Soc. Jpn. 43:82.

31. Watanabe, Y., Umekawa, M., and Nishimura, S. 1978. The causal pathogen of Alternaria black spot of strawberry. (In Japanese.) (Abstr.) Ann. Phytopathol. Soc. Jpn. 44:363.

32. Wattad, C., Dinoor, A., and Prusky, D. 1994. Purification of pectate lyase produced by Colletotrichum gloeosporioides and its inhibition by epicatechin: A possible factor involved in the resistance of unripe avocado fruits to anthracnose. Mol. Plant-Microbe Interact. 7:293-297.

33. Yamamoto, M., Nishimura, S., Kohmoto, K., and Otani, H. 1984. Studies on host-specific AF-toxins produced by Alternaria alternata strawberry pathotype causing Alternaria black spot of strawberry. (2) Role of toxins in pathogenesis. Ann. Phytopathol. Soc. Jpn. 50:610-619.

34. Yamamoto, Y., Oku, H., Shiraishi, T., Ouchi, S., and Koshizawa, K. 1986. Non-specific induction of pisatin and local resistance in pea leaves by elicitor form Mycosphaerella pinodes, M. melonis and M. ligulicola and the effect of suppressor from M. pinodes. J. Phytopathol. 117:136-143.

35. Yoder, O. C., and Scheffer, R. P. 1969. Role of toxin in early interactions of Helminthosporium victoriae with susceptible and resistant oat tissues. Phytopathology 59:1954-1959. 